\title{
Resenha: \\ The dimensions of hegemony: Language, Culture and Politics in Revolutionary Russia
}

\author{
Cristiane Lenz ${ }^{1}$
}

Os estudos marxistas que hoje problematizam a língua em sua relação com a história e com a ideologia devem seus avanços em grande parte aos estudos desenvolvidos na Rússia ao longo da primeira metade do século XX. As questões de linguagem são problematizadas sob diferentes óticas na Rússia pós-revolução, na medida em que novas demandas se instauram no seio da sociedade russa soviética, conforme nos ensina o especialista em Teoria cultural e História de intelectuais russos e soviéticos, Craig Brandist, em Dimensions of hegemony (2015)2.

Esta obra divide-se em oito capítulos que dissertam sobre os paradigmas das ciências da linguagem no período revolucionário na Rússia, e, principalmente, dissertam sobre como as demandas da sociedade russa pós-revolução, tais como definidas por Vladimir Lênin, fomentaram a busca por desenvolvimentos práticos e teóricos que as respondessem. Essas demandas surgem a partir do que é definido como "hegemonia do proletariado", considerando que isso significa, para os bolcheviques, "o poder à classe trabalhadora", de acordo com as palavras de Zinov'ev (BRANDIST, 2015, p. 9). No entanto, o próprio conceito de hegemonia era um debate constante entre os intelectuais. Assim, para compreender a construção do pensamento linguístico marxista na Rússia soviética nos anos que sucederam a revolução, é preciso conhecer os movimentos do pensamento intelectual paralelamente à história das lutas na nação russa. Esta leitura nos é ofertada em Dimensions of hegemony, cujo objetivo é assim descrito por Brandist (2015, p. 17):

The current study traces the idea of hegemony in the political and intellectual debates in the last years of the Russian Empire, the various dimensions that were developed throughout the first decade following the Revolution and the way in which it disappeared from Soviet-theoretical discourse in the 1930s. It is an intellectual history that traces the relationship between ideas about hegemony and about language $\left[\ldots .{ }^{3}\right.$

Brandist observa que o proletariado russo era composto por trabalhadores frequentemente semi-alfabetizados, e os camponeses eram largamente analfabetos, além de que sua fala era muito distante do padrão da língua russa. Então, uma das principais preocupações de Lênin era que os proletários e os camponeses tivessem condições intelectuais de participar da vida social e política e exercer a prática democrática. Assim, o próprio conceito de hegemonia de Lênin carrega as necessidades da sociedade russa em criar tais condições:

1 Mestra em Letras pela Universidade Federal do Rio Grande do Sul.

2 Tradução sob responsabilidade da autora: "Dimensões da hegemonia."

3 Tradução sob responsabilidade da autora: "O presente estudo traça a idéia de hegemonia nos debates políticos e intelectuais dos últimos anos do Império Russo, as várias dimensões que foram desenvolvidas ao longo da primeira década após a Revolução e o modo como este termo desapareceu do discurso teórico soviético na década de 1930. É uma história intelectual que traça a relação entre ideias sobre hegemonia e sobre a linguagem.” 
The hegemony of the working class is the political influence that class (and its representatives) exercises upon other sections of the population by helping them to purge their democracy (where there is democracy) of undemocratic admixtures, by criticising the narrowness and short-sightedness of all burgeios democracy, by carrying on the struggle against Cadetism (meaning the corrupting ideological content of the speeches and policy of the liberals) [...] (LENIN, 1974, apud BRANDIST, 2015, p. 29) 4

Nesse sentido, a hegemonia do proletariado não se reduzia a um senso ordinário de "liderança", mas tinha o alcance da perspectiva de manter a aliança entre o proletariado e o campesinato (smyshka) e criar condições para que estas classes exercessem a democracia. Uma prática muito importante que deriva do próprio conceito de hegemonia é a criação do Instituto da Palavra Viva (Institut zhivogo slova) em Petrogrado ${ }^{6}$. Segundo Brandist (op. cit., p. 91), os objetivos do instituto seriam os seguintes:

1) Scientific-practical working out of questions relating to the realm of the living Word and associated disciplines; 2) the preparation of specialists of the living word in pedagogical, socio-political and artistic fields; and 3) the distribution and popularization of knowledge and mastery of the field of the living word. ${ }^{7}$

Para os líderes bolcheviques que trabalhavam pelas Organizações da Cultura e do Esclarecimento Proletário ${ }^{8}$ (Proletarskie kul'turno-prosvetitel'nye organizatsii, p. 86), o Prolekul't, tais como o seu primeiro comissário, Lunacharskii, e Nadezhda Krupaskaia, esposa de Lênin, a democracia e a palavra viva eram inseparáveis na medida em que o domínio da língua por todo o povo russo era condição para o processo de democratização.

O conceito de palavra viva era, portanto, essencial para o Prolekul't. Nesse sentido, havia a preocupação com várias formas de "cultural integration" (2015, p. 84), ou seja, a integração cultural, conforme expressão do poeta Viacheslav Ivanov, que defendia que no interior da luta de classes esta demanda estava implícita. Assim, eram criadas as condições para o desenvolvimento de atividades culturais, tais como o teatro, os estudos de oratória, estudos de poesia e declamação, estudos sobre arte, além da manutenção

4 Tradução sob responsabilidade da autora: "A hegemonia da classe trabalhadora é a influência política que a classe (e seus representantes) exercem sobre outras camadas da população, ajudando-os a purgar sua democracia (onde há democracia) de misturas antidemocráticas, criticando a estreiteza e miopia de de toda a democracia burguesa, conduzindo a luta contra o cadetismo (ou seja, o conteúdo ideológico corruptor dos discursos e da política dos liberais)."

5 Smichka seria a denominação para a aliança entre o proletariado e o campesinato. Ao longo do período revolucionário, essa aliança seria objeto de debate e preocupação, na medida em que a sociedade russa era composta predominantemente por camponeses: "The need to maintain proletarian hegemony in a predominantly peasant society and, further, in a multhi-ethnic, multi-lingual, multi-faith state in which the legacy of imperialism needed to be addressed, was fundamental in shaping the agendas of early Soviet scholars." (BRANDIST, 2015, p. 221) Tradução sob responsabilidade da autora: "A necessidade de manter a hegemonia proletária numa sociedade predominantemente camponesa e, além disso, num estado multiétnico, multilingue e multi-religioso, em que o legado do imperialismo precisava ser considerado, era fundamental na formação dos objetivos dos primeiros intelectuais soviéticos."

6 Trata-se da cidade que hoje se chama São Petersburgo, e que também já foi chamada Leningrado.

7 Tradução sob responsabilidade da autora: “1) o tratamento científico e prático de questões relativas ao domínio da palavra viva e a disciplinas associadas; 2) a preparação de especialistas da palavra viva nos campos da pedagógicos, sociopolíticos e artísticos; 3) a distribuição e a popularização do conhecimento e domínio do campo da palavra viva."

8 Esta expressão é uma tradução sob responsabilidade da autora do inglês Proletarian Cultural and Enlightenment Organisations. 
de clubes, bibliotecas, escolas partidárias, educação de adultos e cabanas de leitura (village reading-huts, p. 88 ).

A própria formação do Exército Vermelho, cujo motivo de criação recaía principalmente na problemática da existência de uma minoria proletária em comparação com a maioria camponesa, teve por base a preocupação em fazer com que o soldado trabalhador fosse consciente de si e das necessidades de sua nação, substituindo, segundo Trotsky, o "instinto de rebanho cego" (blind herd instinct) do exército tsarista, pelo "cimento psicológico" (psychological cement) que pudesse criar um exército novo e consciente.

Trotsky made it clear that the aim was to form a peasant and worker soldier who is aware of himself as a human personality, with a right to respect, but also feels that he is part of the working class of republican Russia and will be prepared unquestioningly to lay down his life for this Republican Soviet Russa. (BRANDIST, 2015, p. 107) ${ }^{9}$

Todas estas práticas residiam na preocupação do que era definido como a questão nacional, que consistia no reconhecimento de que era preciso que o proletariado russo fosse capaz de conquistar e manter a liderança sobre uma variedade de grupos étnicos e culturais, para os quais faltava um senso de identidade e uma consciência sobre sua própria cultura, além de uma forma padrão de língua, conforme já mencionamos.

Em meio a este contexto, os paradigmas das ciências da linguagem se dividiam entre perspectivas formalistas-positivistas, na Escola de Moscou, e psicologistas, na Escola de São Petersburgo. Brandist observa, no entanto, que nenhuma era capaz de aceitar ou rejeitar completamente as concepções de Durkheim ${ }^{10}$ ou de Saussure.

$\mathrm{Na}$ segunda metade dos anos 1920, o ILIaZV ${ }^{11}$ (Instituto de Pesquisa Científica em História Comparada da Literatura e das Línguas do Oeste e do Leste), em Leningrado, trabalhou na evolução de questões gerais de estudos de língua e literatura e levantou comentários sobre linguagem nos clássicos marxistas. Assim, o Instituto tinha por objetivos:

1) Problems of international and intra-national linguistic and literary exchange on the basis of the socio-economic, political, and general cultural interaction of peoples and countries. 2) The interaction of linguistic units (national and class languages, ethnic and social dialects and so on.) 3) International literary exchange in connection with the social development of peoples and countries that are in literary interaction. ${ }^{12}$ (op. cit., p. 130)

9 Tradução sob responsabilidade da autora: "Trotsky tornou claro que o objetivo era formar um soldado camponês e trabalhador que fosse consciente dele mesmo como uma personalidade humana, com o direito ao respeito, mas que se sentisse parte da classe trabalhadora da Rússia republicana e que estivesse preparado para, inquestionavelmente, dar sua vida pela Rússia soviética republicana."

10 Era comum a leitura de que a definição saussureana de langue como um fato social correspondesse à ótica de Émile Durkheim de que "[...] a social fact is 'any way of acting, whether fixed or not, capable of exerting over the individual na external constraint' [...]" (DURKHEIM, 1982, apud BRANDIST, 2015, p. 128) Tradução sob responsabilidade da autora: "um fato social é 'qualquer forma de agir, seja fixa ou não, capaz de exercer sobre o indivíduo uma limitação externa'."

11 Em nota sobre o ILIaZV, Brandist destaca que "Like many institutes, it changed its name and form a number of times. It was the Aleksandr Veselovskii Institute of Language Studies and the History of Literature [Institut iazykovedeniia i istorii literatury im.ak. A.N. Veselovskogo] 1921-3, ILIaZV, 1923-30 and The State Institute for Discursive Culture [Gosudarstvennyi institut rechevoi kul'tury, (girk) 1930-3].” (2015, p. 94) Tradução sob responsabilidade da autora: "Como muitos institutos, [ILIaZV] trocou de nome e formato algumas vezes. Foi Aleksandr Veselovskii Instituto de Estudos da Língua e da História da Literatura de 1921 a 1923, ILIaZV de 1923 a 1930, e Instituto do Estado de Cultura Discursiva entre 1930 e 1933."

12 Tradução sob responsabilidade da autora: "1) Problemas de intercâmbio lingusítico e literário internacional e intra-nacional com base na interação sócio-econômica, política e cultural dos povos e países. 2) A 
Este grupo de estudos observou que a influência mútua da literatura e das belas artes entre os países seria resultante de uma convergência das estruturas sociais de tais países. O trabalho de Wilhelm Humboldt foi influente na medida em que propunha que a forma linguística e o seu conceito deveriam ser tratados inseparavelmente. Os trabalhos de Lev Iakubinskii observaram os aspectos de constituição do diálogo, desde as expressões dêiticas, gestos faciais e corporais, elipses, além de como as características do grupo social a que o falante pertence emergem na interação discursiva.

Entre os trabalhos mais conhecidos resultantes do ILIaZV encontra-se o de Valentin Voloshinov. O linguista desenvolveu seus estudos no sentido de contemplar o diálogo cotidiano e a poesia como dois tipos de enunciados, baseando-se no modelo de atos de fala de Karl Bühler. O sentido de um signo, assim, depende de uma configuração entre o falante, o ouvinte e o objeto sobre o qual se fala, enquanto que, na poesia, apenas um contexto linguístico é oferecido ao falante ou ao ouvinte.

É importante destacar que, neste período, as concepções saussureanas de língua estavam sendo lidas e criticadas. A obra de Voloshinov, Marxism and Philosophy of Language $^{13}$, faz uma crítica à teoria do signo linguístico. Para ele, o sistema abstrato, associado à langue saussureana, corresponde apenas a uma necessidade da filologia de estudar línguas mortas e de ensinar a língua, e isso se opõe à interação dinâmica que é a realidade da língua viva. Em oposição a isso, Brandist (2015, p. 132 e 133) nos explica o seguinte:

Here [in Marxism and Philosophy of Language], Voloshinov presented an account of language as social interaction in which the specific meaning that a word acquires in a concrete dialogue is shaped by the respective positions the interlocutors occupy in the social structure. ${ }^{14}$

Ao mesmo tempo que faz essa crítica, Voloshinov também se preocupa em observar os trabalhos do que chama "individualistic subjectivism" (2015, p. 134) - individualismo subjetivista - de Benedetto Croce e Karl Vossler. Ainda que tal observação seja crítica e aponte os aspectos inaceitáveis de tal corrente de pensamento, as considerações são de tom mais respeitável, segundo observa Brandist.

Iakubinskii compartilha a visão de Voloshinov em seu trabalho de 1929 e expõe uma crítica a Saussure, fazendo a seguinte consideração: "He [Saussure] thinks that the only possible knowledge about language is by means of reflection, contemplation from the side, forgetting about the subject of language, about the collective." ${ }^{5}$ (IAKUBINSKII, 1966, p. 81, apud BRANDIST, 2015, p. 134)

Os trabalhos de Iakubinskii são fundamentais para compreendermos como a história da linguística enquanto ciência se desenvolve em relação de constituição com a história da revolução de 1917 e dos anos subsequentes. Iakubinskii discute as consequências linguísticas para o campesinato do avanço do mercado nacional, ou seja, o avanço do

interação de unidades lingüísticas (línguas nacionais e de classe, dialetos étnicos e sociais, entre outras). 3) Intercâmbio literário internacional em conexão com o desenvolvimento social de povos e países que estão em interação literária."

13 É importante ressaltar que esta obra, Marxismo e filosofia da linguagem, foi publicada originalmente sob a autoria de Valentin Voloshinov.

14 Tradução sob responsabilidade da autora: "Aqui [em Marxismo e Filosofia da Linguagem], Voloshinov apresentou um relato da linguagem como interação social na qual o sentido específico que uma palavra adquire em um diálogo concreto é moldado pelas posições respectivas que os interlocutores ocupam na estrutura social."

15 Tradução sob responsabilidade da autora: "Ele [Saussure] acha que o único conhecimento possível sobre a língua é por meio da reflexão, da contemplação lateral, esquecendo o sujeito da língua e da coletividade." 
capitalismo fez com que os camponeses tivessem que optar pela língua urbana, em detrimento do seu dialeto. $\mathrm{O}$ autor chama a atenção para o fato de que esses efeitos não foram lineares ou desprovidos de luta e resistência por parte do campesinato, na medida em que seu dialeto significava também sua cultura e sua religião (IAKUBINSKII, 1930, apud BRANDIST, 2015). Os estudos de Iakubinskii foram inspiradores para outros linguistas, como Zhirmunskii, que argumentou que a língua nacional seria o dialeto social da classe dominante.

Brandist observa que tais estudos evidenciam as distorções de Stalin em suas considerações sobre o que seria uma nação. Vejamos a sua concepção de nacionalidade: "[...] a historically constituted, stable community of people, formed on the basis of a common language, territory, economic life, and psychological make-up manifested in a common culture." ${ }^{\prime 6}$ (STALIN, 1953, p. 307, apud BRANDIST, 2015, p. 103). Tal noção realiza um apagamento da diversidade cultural, linguística e étnica, e propõe um sentido ilusório de unidade nacional. Conforme Brandist nos mostra, os estudos da linguagem se situavam em relação à questão nacional tal como delineada nos primeiros anos pósrevolução, ou seja, considerando a variedade de grupos étnicos e culturais que formavam a nação russa, ao contrário do que propõe Stalin.

Enquanto os estudos em Leningrado ancoravam-se em visões psicologistas, e se encaminhavam para uma perspectiva sociológica, com forte crítica à teoria saussureana, os estudos em Moscou se situavam em uma corrente formalista, que ofereceu à langue uma recepção mais positiva. No interior deste Círculo, Nikolai Iakoklev desenvolveu tabelas fonéticas e fórmulas matemáticas para a construção de alfabetos. Mikhail Peterson trabalhava na criação de leis gerais, com influência de Émile Durkheim e Antoine Meillet. Ao mesmo tempo, Anton Marty faz uma crítica ao psicologismo ao colocar a língua em um patamar instrumental, e não como uma construção da mente, criticando diretamente os estudos de Wundt, Croce e Vossler. Além disso, os estudos de Rozaliia Shor se situam no Círculo de Moscou, e representam uma perspectiva altamente positiva da teoria saussureana e uma forte crítica aos estudos de Vossler e Croce.

A partir da leitura de Brandist (2015), compreendemos que é no interior do Círculo de Moscou que Polivanov, aluno de Baudouin de Courtenay, poderá propor um programa de análise sociolinguística bastante inovador, que contemplava, em termos gerais, a combinação dos estudos da linguagem com o marxismo, um estudo das conexões entre fenômenos linguísticos e socioeconômicos e uma análise da evolução da língua sob o prisma da história da cultura. Na perspectiva de Brandist (op. cit., p. 148): "This sociolinguistic programme was nothing but path-breaking, but it unfortunately appeared just as the socio-political situation and institutional structures were changing fundamentally." 17

Além do desenvolvimento dos estudos da linguagem nos Círculos de Leningrado e de Moscou, os estudos de Nikolai Marr têm amplo destaque na obra de Brandist na medida em que se situam, de alguma forma, consonantes com a perspectiva stalinista de língua nacional, e também porque, de acordo com Brandist: "Marr's work stretches across all the periods discussed in this book, emerging from the old schools of philology

16 Tradução sob responsabilidade da autora: "Uma comunidade de pessoas historicamente constituída e estável, formada nas bases de uma língua, um território, uma economia e uma composição psicológica comuns, manifestados em uma cultura comum."

17 Tradução sob responsabilidade da autora: "Este programa não era senão inovador, mas infelizmente aparecia justamente quando a situação sociopolítica e as estruturas institucionais estavam mudando fundamentalmente." 


\section{Conexão Letras}

that were concerned wih the remote past of various languages, and with etymological questions as a primary focus." ${ }^{18}$ (op. cit., p. 193) Segundo Brandist, enquanto os estudos sociológicos da linguagem tinham como preocupação a hegemonia do proletariado e a questão nacional da língua em relação à variedade étnica e cultural, Marr considerava que o próprio movimento histórico conduziria à fusão dos diversos sistemas de línguas em uma única língua.

Finalmente, é importante compreender que, conforme mencionamos no início deste texto, o conceito de hegemonia do proletariado foi fundamental para a forma como os estudos da linguagem se desenvolveram na Rússia soviética pós-revolução e como as próprias instituições foram organizadas no sentido de privilegiar a emancipação do povo russo através da alfabetização e do conhecimento. É notável a criação de organizações de estudo que se destinavam tanto aos trabalhadores e camponeses quanto aos linguistas e demais pensadores. No entanto, o capítulo sete da obra de Brandist situa o declínio do conceito de hegemonia. A conjuntura econômica se modificou bastante a partir da criação do "first Five Year Plan" (2015, p. 176) (primeiro Plano dos Cinco Anos), estabelecido por Stalin. Segundo Brandist, os trabalhadores perderam as conquistas da NEP e ficaram totalmente subordinados ao processo de produção. Nessas condições, a luta de classes não se sustentava e, consequentemente, a aliança entre camponeses e trabalhadores smychka - não consistia mais em um centro de atenção. Assim, o conceito de hegemonia do proletariado perde sua força como paradigma condutor da práxis.

A partir dos destaques propostos neste espaço, procuramos elucidar minimamente alguns focos de atenção que consideramos fundamentais na obra de Brandist. No entanto, é preciso compreender a dimensão deste estudo, que constrói uma narrativa histórica e científica acerca da linguística na Rússia soviética no período pós-revolução. Esta obra coloca condições para que os atuais estudos materialistas da linguagem compreendam suas bases, na medida em que permite compreender de que forma a perspectiva marxista da linguagem na Rússia tem sua origem no interior da Revolução.

\section{Referências}

BRANDIST, C. The dimensions of Hegemony: language, culture and politics in revolutionary Russia. Brill: Boston, 2015.

Recebido em: 23/04/2018 Aceito em: 12/06/2018

18 Tradução sob responsabilidade da autora: “O trabalho de Marr se estende por todos os períodos discutidos neste livro, emergindo das antigas escolas de filologia que estavam preocupadas com o passado remoto de várias línguas e com questões etimológicas como foco principal." 